
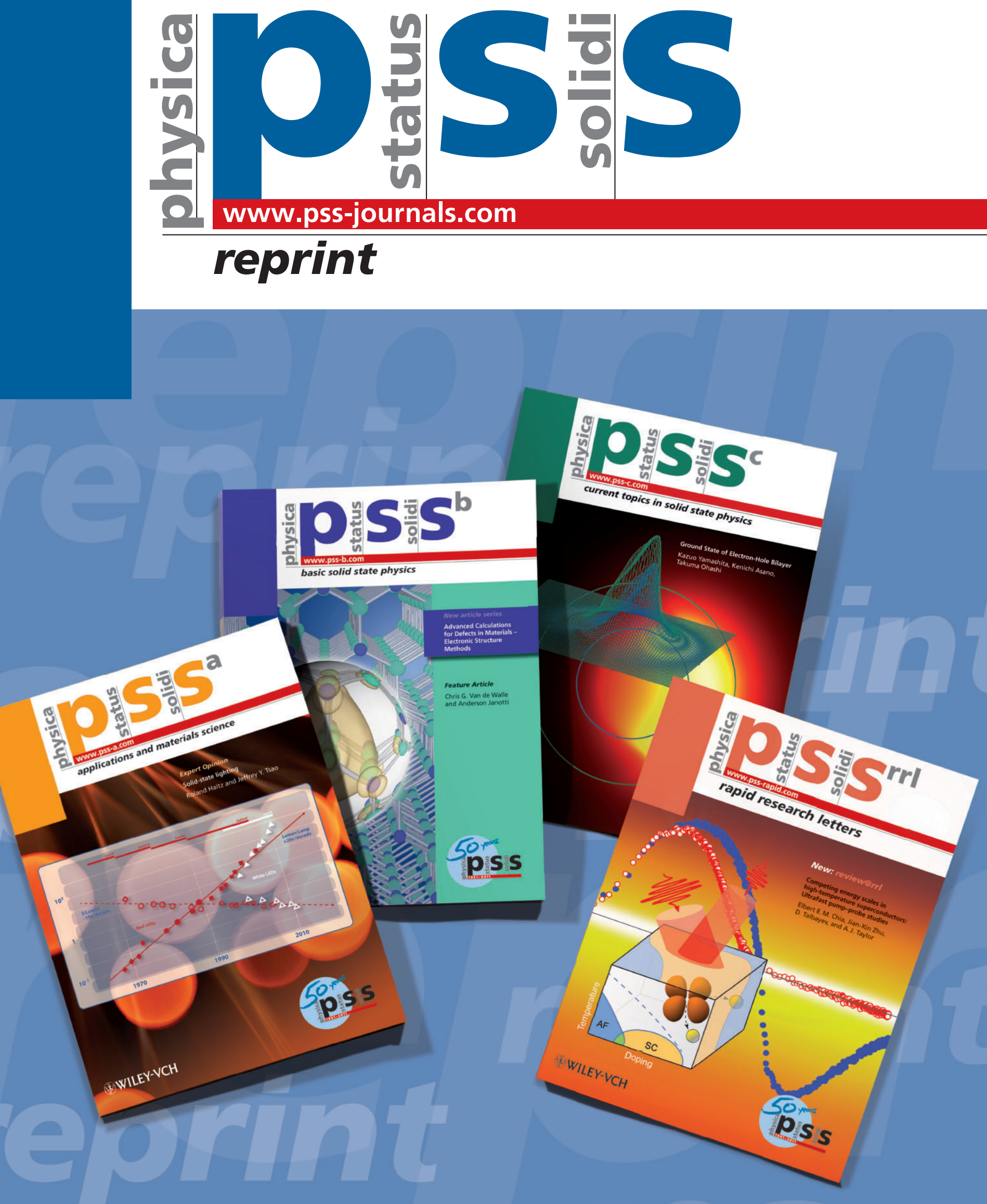


\section{Lattice tilt, concentration, and relaxation degree of partly relaxed InGaAs/GaAs structures}

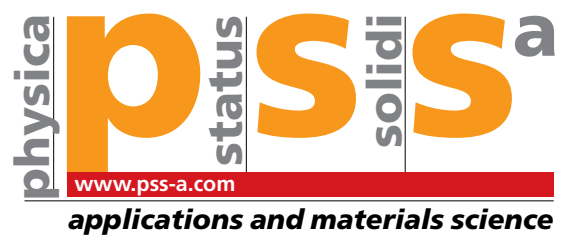

\section{A. Benediktovitch ${ }^{*, 1}$, F. Rinaldi ${ }^{2}$, S. Menzel ${ }^{2}$, K. Saito ${ }^{3}$, T. Ulyanenkova ${ }^{4}$, T. Baumbach ${ }^{4}$, I. D. Feranchuk', and A. Ulyanenkov ${ }^{5}$}

${ }^{1}$ Department of Theoretical Physics, Belarusian State University, 4 Nezavisimosti av., 220030 Minsk, Republic of Belarus

${ }^{2}$ University of Ulm, Institut für Optoelektronik, Albert-Einstein-Allee 45, $89081 \mathrm{Ulm}$, Germany

${ }^{3}$ Bruker AXS K.K., 3-9 Moriya, Kanagawa, 221-0022 Yokohama, Japan

${ }^{4}$ University Karlsruhe, Engesserstr. 15, 76131 Karlsruhe, Germany

${ }^{5}$ Bruker AXS GmbH, Östl. Rheinbrückenstr. 49, 76187 Karlsruhe, Germany

Received 28 November 2010, accepted 27 June 2011

Published online 7 October 2011

Keywords high-resolution reciprocal space mapping, partly relaxed semiconductor systems, misfit dislocations

*Corresponding author: e-mail aib@tut.by, Phone: +375-17-2265700, Fax: +375-17-2134420

The series of samples is investigated to verify the validity of the scattering theory within the layers of different relaxation degree. The samples composed of $\mathrm{In}_{0.06} \mathrm{Ga}_{0.94}$ As layer of different thicknesses on GaAs [001] substrates were grown using MBE technique. The symmetric and asymmetric reciprocal space maps (RSM) were measured and simulated for the samples with the fully coherent layer, in the vicinity of the critical thickness of relaxation, and with the fully relaxed layer. The crystallographic layer miscuts, indium concentrations, the relaxation degrees, and density of dislocations have been precisely evaluated.
1 Introduction The electronic properties of semiconductor devices depend essentially on the designed strain profile, relaxation status of the crystallographic lattice, and various structure imperfections, viz. lattice tilts, dislocations, defects, etc. The proper characterization of these physical parameters is a pre-condition of the successful sample growth. The relaxation process of the crystallographic lattice plays a key role in the formation of the final structure of semiconductor device. In many cases, the relaxation is a part of the design, for example, in SiGe virtual substrates, buffer layers in $\mathrm{GaN}$ technology, etc. The high-resolution X-ray reciprocal space mapping (RSM) is an universal technique to simultaneously and consistently characterize all the mentioned above parameters. The X-ray scattering process is, however, not straightforward in this case [1], due to the overlapping of the effects of the dynamical X-ray scattering, the lateral crystallographic mismatches, and the defects initiated by the relaxation process. Therefore, the accurate data evaluation requires the precise accounting of the numerous effects, including dynamical X-ray scattering, lattice tilts, presence of the dislocation networks, etc. In this work, the details and peculiarities of the typical evaluation process are described on the example of a single $\mathrm{In}_{0.06} \mathrm{Ga}_{0.94} \mathrm{As}$ layer in the different states, pseudomorphic, partly and fully relaxed, on the GaAs substrate. The dynamical diffraction theory used accounts the scattering from mismatched lattice at the boundary interface between the layer and the substrate. The use of RSMs for the sample evaluation involves the calculation of the crystallographic lattice tilts prior the numerical estimate of the layer relaxation degree and indium concentration from the peak positions. The broadening of the diffraction spots on the RSMs is simulated [2] by the presence of the dislocations, which are caused by the relaxation process occurring in the layer. All the mentioned effects, being accurately accounted in data analysis, permit to consistently and comprehensively characterize the semiconductor samples.

2 Sample growth and measurements The measurements were done by using D8 DISCOVER diffractometer (Bruker AXS). In the high-resolution mode, the $\mathrm{X}$-rays are converged and monochromatized by the Göbel 
mirror and Ge (400) asymmetric channel cut monochromator. The monochromator reduces both wavelength and angular spreads to $\Delta \lambda / \lambda=5.9 \times 10^{-3}$ and $\Delta \theta / \theta=$ $4.0 \times 10^{-3}$ degrees, respectively, for $\mathrm{Cu} \mathrm{K} \alpha_{1}$ radiation. The scattered X-ray intensity is collected by a PSD detector (VÅNTEC-1, Bruker AXS), which covers up to $12^{\circ}$ in $2 \theta$ area with a minimum angular resolution of about $5.0 \times 10^{-3}$ degrees determined by the PSD pixel size.

3 Theory Reciprocal space maps contain lots of information about the structure of the thin film and the substrate. Some information can be extracted by means of simple operations with RSM like the peak position analysis, however, to get precise information about the structure a rigorous analysis is demanded. Below the information obtained from evaluation is considered in the order of the complexity of used methods.

3.1 3D miscut Positions of the substrate and the layer peaks are directly connected to corresponding reciprocal lattice vectors. If the crystal lattice is tilted corresponding to the interface the diffraction peaks will be shifted in according to the tilt. This shift depends also on experimental geometry and $(h \mathrm{kl})$ reflection used. From each RSM the projection of the tilt on the diffraction plane can be found and with the help of several RSM measured in several azimuthal directions the 3D miscut orientation and absolute value can be reconstructed, Fig. 1.

3.2 Concentration, relaxation, thickness The peak positions enable to find a magnitude of the reciprocal lattice vector and hence in-plane and out-of-plane lattice period. With the help of the Vegard's law, definition of relaxation and the Hook's law the concentration and relaxation values can be found [3]. For correct evaluation also the values of miscut should be taken into account. The results for the investigated samples are summarized in Table 1, the relaxation value $R$ is defined as:

$$
R=\frac{a_{\|}-a_{\|}^{(\mathrm{s})}}{a_{\|}^{(0)}-a_{\|}^{(\mathrm{s})}},
$$

where $a_{\|}$the is actual in-plane lattice parameter of the film, $a_{\|}^{(0)}$ is the in-plane lattice parameter the film would have in
Table 1 The values of sample layer thickness $t_{1}$, absolute lattice tilt $\delta$, and its orientation $\phi_{0}$ for layer and substrate, concentration $c$ and relaxation degree $R$.

\begin{tabular}{lllllll}
\hline$t_{1}(\mathrm{~nm})$ & $\phi_{0 \mathrm{~s}}\left({ }^{\circ}\right)$ & $\delta_{\mathrm{s}}\left(^{\circ}\right)$ & $\phi_{01}\left(^{\circ}\right)$ & $\delta_{1}\left(^{\circ}\right)$ & $c(\%)$ & $R$ \\
\hline 200 & 271 & 0.033 & 259 & 0.016 & 5.6 & 0.07 \\
400 & 253 & 0.003 & 263 & 0.003 & 5.6 & 0.05 \\
450 & 135 & -0.001 & 135 & 0.001 & 4.4 & 0.02 \\
500 & 62 & 0.01 & 81 & 0.025 & 5.5 & 0.03 \\
550 & 227 & 0.01 & 357 & 0.03 & 5.4 & 0.55 \\
600 & 138 & 0.019 & 301 & 0.029 & 5.8 & 0.65 \\
800 & 293 & 0.018 & 32 & 0.050 & 5.4 & 0.59 \\
1200 & 280 & 0.02 & 354 & 0.035 & 5.5 & 0.73 \\
\hline
\end{tabular}

completely relaxed free-standing state, $a_{\|}^{(\mathrm{s})}$ is the in-plane lattice parameter of the substrate.

The layer parameters extracted from the estimation are used further for calculation of RSMs. There are several approaches to RSM calculation (dynamical diffraction theory for epitaxial structures, diffuse scattering from defects in distorted structures), the comparison of the calculated RSM with the experimental one enables to choose which one is correct and to derive the conclusions about layer microstructure.

3.3 Thin layer microstructure In the frame of dynamical diffraction theory the RSM from partly relaxed structure should look like stripes along the $q_{z}$ direction [1]. This is due to in-plane boundary conditions leading to $q_{\|}$ conservation. However, the actual experimental RSM have such structure only in case of thin pseudomorphic layers. As the relaxation increases the shape of RSM transforms from stripes along the $q_{z}$ direction to spots approximately elliptic in shape and inclined in the direction close to the perpendicular to reciprocal lattice vector. At intermediate stages of relaxation, the transition shapes can be observed [4]. To extract a quantitative data from the RSM taken from the partly relaxed layers the theory describing diffraction on real crystals should be used. There has been a lot of activity in past decades in the area of simulation of diffraction from the real crystals possessing defects. The theoretical approaches usually follow the line of reasoning given by Krivoglaz and Wilkens, see Ref. [5] and references therein, and the most of
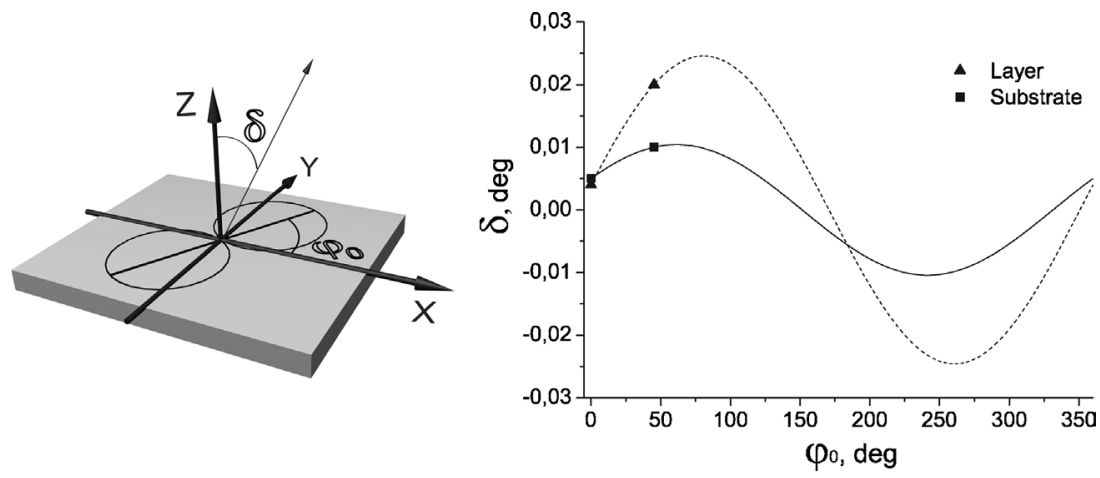

Figure 1 The evaluation of 3D miscut. Left: a sketch of geometry used for 3D miscut evaluation. The curved line represents a polar plot of the cosine function used for the fit of 3D miscut $\delta$ dependence on angle $\phi . X$-axis is directed along [100] direction, $Y$-axis is along [010]. $\phi_{0}$ determines the plane in which the miscut has the maximal value; right: fit of the obtained miscut experimental data. 
the publications are dedicated to the modeling of diffraction from the polycrystals. The diffraction from the relaxed layers has significant differences from that from polycrystals, one of the reason being a different arrangement of the defects. The extension and further development of initial Krivoglaz and Wilkens ideas applied to partly relaxed thin films was carried out in the works of Kaganer. Here, in order to perform the analysis of RSM from partly relaxed structures we follow the approach developed in Ref. [6]. Below the basis of this approach is shortly reminded. The relaxation of the film is assumed to be due to misfit component of the Burgers vector of edge dislocations. Other Burgers vector components are supposed to have positive and negative values with equal probability. For the present structure $60^{\circ}$ dislocations are considered and two systems of them are assumed to lie along [110] and [1-10] directions. Due to the presence of dislocations, the atoms are removed from their regular positions on the value given by sum of displacements fields caused by all dislocations:

$$
\boldsymbol{R}=\boldsymbol{R}_{n}+\sum_{t, \alpha, l} c_{t, \alpha} \boldsymbol{u}^{(l)}\left(\boldsymbol{r}_{\boldsymbol{n}}-\boldsymbol{r}_{\boldsymbol{t}}\right) \boldsymbol{b}_{\alpha}^{(l)}
$$

here $c_{t, \alpha}$ is probability to find the dislocation of family $\alpha$ at site $\boldsymbol{r}_{t}$, the $\boldsymbol{u}^{(l)}$ is the displacement caused by $l$-th projection of the Burgers vector $\boldsymbol{b}_{\alpha}$ of dislocation of family $\alpha$. In the framework of kinematical diffraction theory the expression for diffracted X-ray intensity was shown to have the following form:

$$
I(\boldsymbol{q})=\int \mathrm{d}^{3} \boldsymbol{r} \mathrm{d}^{3} \boldsymbol{r}^{\prime} \mathrm{e}^{-i \boldsymbol{q}\left(\boldsymbol{r}-\boldsymbol{r}^{\prime}\right)} \mathrm{e}^{-T\left(\boldsymbol{r}, \boldsymbol{r}^{\prime}\right)},
$$

where

$$
T\left(\boldsymbol{r}, \boldsymbol{r}^{\prime}\right)=\sum_{\alpha} \rho_{\alpha} \int \mathrm{d}^{3} \boldsymbol{r}^{\prime \prime} \Phi\left(\boldsymbol{r}, \boldsymbol{r}^{\prime}, \boldsymbol{r}^{\prime \prime}\right),
$$

is a correlation function which for Poisson statistics of dislocation occupation probability takes the form Ref. [6]

$$
\Phi\left(\boldsymbol{r}, \boldsymbol{r}^{\prime}, \boldsymbol{r}^{\prime \prime}\right)=1-\mathrm{e}^{-i \boldsymbol{h}\left(\boldsymbol{u}\left(\boldsymbol{r}-\boldsymbol{r}^{\prime \prime}\right)-\boldsymbol{u}\left(\boldsymbol{r}^{\prime}-\boldsymbol{r}^{\prime \prime}\right)\right)},
$$

$\boldsymbol{q}$ is deviation of transferred wave vector $\boldsymbol{Q}$ from reciprocal lattice vector $\boldsymbol{h}$ of reflex considered. The intensity distribution (3) contains diffuse and coherent ( $\delta$-function) parts, the latter being damped by static Debye-Waller factor that has the order of $\mathrm{e}^{-\rho d}$. In general case the calculation according to given formulas is not straightforward, however, for limiting cases closed analytical expressions can be obtained. Namely, if $\rho_{\alpha} d \gg 1$, where $d$ is film thickness, it was shown in Ref. [6] that the main contribution to the integral in (3) comes from the area far from the dislocations core where the exponent in (3) can be expanded is series to the second order. Then (4) and (3) can be evaluated analytically and the intensity distribution has the form close to anisotropic Gaussian distribution:

$$
I\left(q_{x}, q_{z}\right)=\pi \int_{0}^{d} \frac{\mathrm{d} z}{\operatorname{Det}\left[w_{i j}(z)\right]} \mathrm{e}^{-\left(\left(w_{i j}^{-1}(z)\left(q_{i}-q_{i}^{(0)}\right)\left(q_{j}-q_{j}^{(0)}\right)\right) / 4\right)},
$$

here $i$ and $j$ are used to denote array of index $x, z$, matrix $w_{i j}$ is composed of derivative of elastic fields displacements:

$$
w_{i j}(z)=\sum_{\alpha} \rho_{\alpha} \int_{-\infty}^{\infty} \mathrm{d} x^{\prime} Q_{k} \frac{\partial u_{k}^{l}(\boldsymbol{r})}{\partial x_{i}} b_{l}^{\alpha} Q_{m} \frac{\partial u_{m}^{p}(\boldsymbol{r})}{\partial x_{j}} b_{p}^{\alpha},
$$

and can be evaluated to analytical expression when the form of elastic fields in solid films [6] are substituted. As seen from (6) the maximum of diffuse scattering is shifted from bulk value on $b^{0}$. This shift is calculated from first-order derivatives of displacement fields and the value is:

$$
q_{x}^{(0)}=\rho Q_{x} b_{x}, \quad q_{z}^{(0)}=-\frac{2 v}{1-v} \rho Q_{z} b_{x} .
$$

This values of peak shift can be obtained from consideration of homogeneously averaged deformations accommodated by dislocations's Burgers vector.

4 Discussion The dynamical diffraction theory and the approach of Kaganer were used to interpret RSMs measured from the samples of different thickness. RSMs from samples of thickness in the range 200-450 $\mathrm{nm}$ agree in the shape with the dynamical diffraction theory predictions
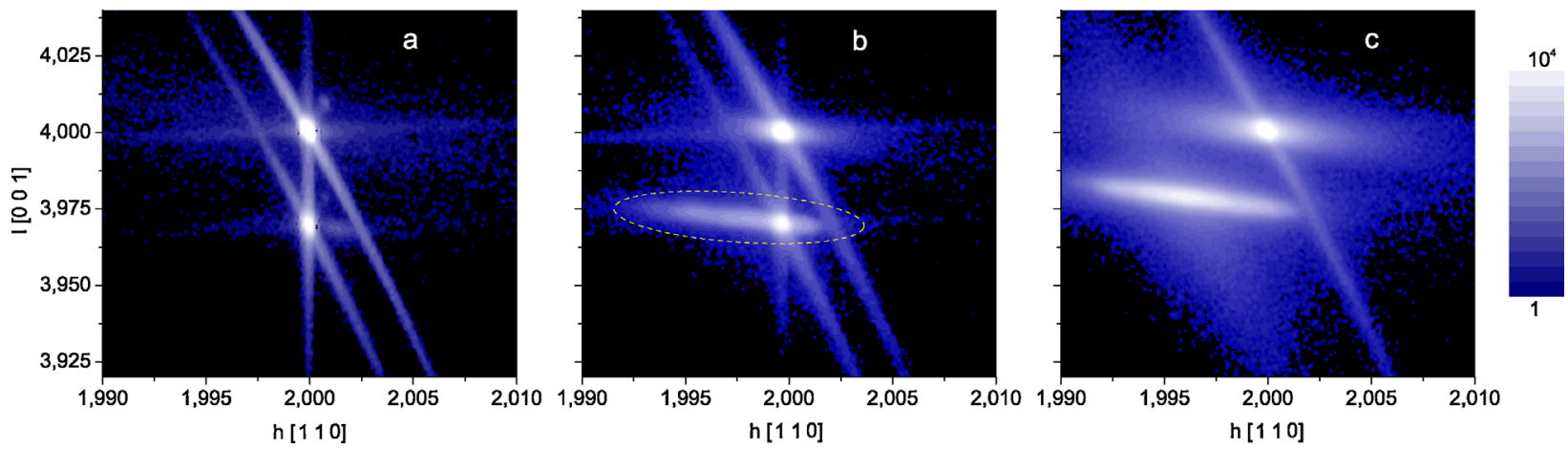

Figure 2 (online color at: www.pss-a.com) Experimental RSMs for the samples of thickness: $200 \mathrm{~nm}$ (a), $500 \mathrm{~nm}$ (b), $1200 \mathrm{~nm}$ (c). In the case (b), the onset of the diffuse scattering is marked with dashed line. 

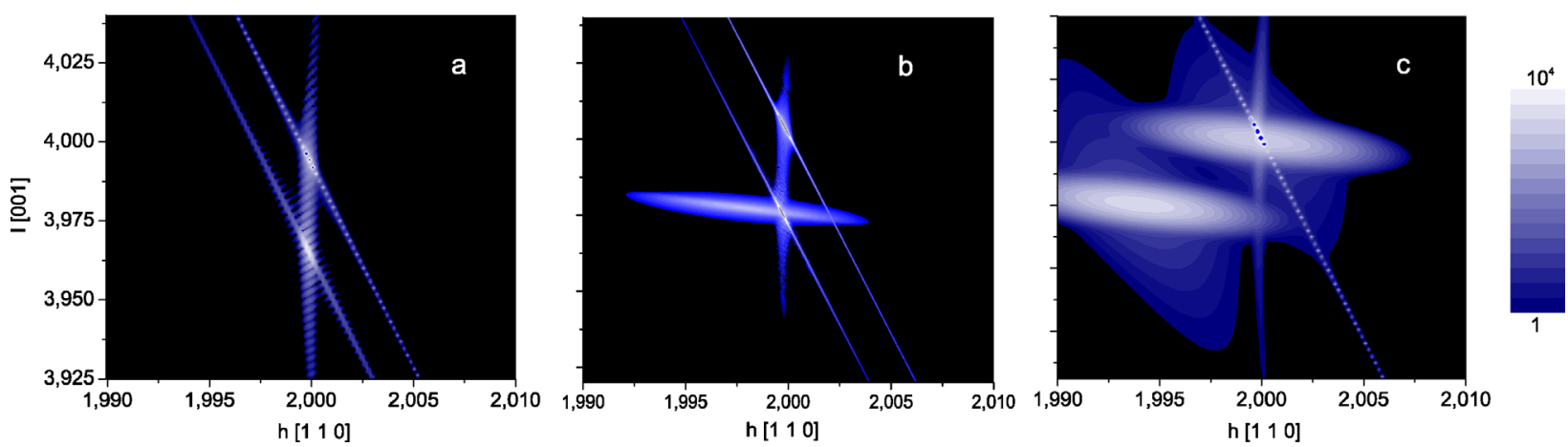

Figure 3 (online color at: www.pss-a.com)Calculated RSMs for the samples of thickness: $200 \mathrm{~nm}$ (a), $500 \mathrm{~nm}$ (b), $1200 \mathrm{~nm}$ (c). The map (a) is calculated on the basis of dynamical diffraction theory, (c) on the basis of Kaganer's approach, in (b) both are used.

for pseudomorphic structures (Figs. 2a and 3a). There is a single stripe along $q_{z}$ direction, and thin film thickness oscillations can be observed. The inclined stripes are due to diffractometer optics and do not carry information about the investigated sample. The simulation according to dynamical diffraction theory, with the parameters obtained from the peak positions as described in the Section 3.2, show agreement with the experimental data. If incoherent scattering was included in the simulation, we would obtain wide intensity spread in the $q_{x}$ direction that is not observed in the experimental data. Hence, it can be concluded that the diffraction is determined by the coherent scattering processes.

In the case of thick samples $(600-1200 \mathrm{~nm})$ the RSMs have the shape of elliptic spots with the semi-minor axis being approximately parallel to the diffraction vector $\boldsymbol{Q}$, Fig. 2c. This kind of shape is predicted in Kaganer's theory (6), and supports the applicability of the approach. The center of diffraction spots are connected via (8) with the density of misfit dislocations, the obtained dislocation density was used for RSM simulation, Fig. 3c.

The RSM for the sample of $500 \mathrm{~nm}$ thickness shows the transitional behavior, Fig. $2 \mathrm{~b}$. There can be clearly observed a stripe along $q_{z}$ axis which gives the evidence of the presence of coherent part of diffraction. In addition one can notice an elongated spot of diffracted intensity close to the position of layer's maximum. It can be interpreted as sign of diffuse scattering onset, the center of the elongated spot being related to the misfit dislocation density. The theory presented in Section 3 is not directly applicable to this case since the quantity $\rho d$ is of order of unity. However, we used the sum of coherent intensity calculated according to the dynamical diffraction theory and diffuse contribution according to the formulas of Section 3. The calculated RSM shape is satisfactorily close to the experimental one, Fig. $3 b$.

The X-ray measurements from the samples of different thickness clearly demonstrate how the film changes the state from pseudomorphic to partly relaxed. The relation between thickness of the film and its relaxation is a result of numerous complex microscopic processes of dislocation propagation, nucleation, multiplication, see Ref. [7] and references there.
The energetic balance theory of Mathews and Blakeslee for critical thickness of relaxation onset cannot describe our experimental data since it was derived for propagation of a single dislocation, while the X-ray diffraction being sensitive to the collective effects within the crystal. However, the connection between the thickness and relaxation following from Mathews and Blakeslee approach [8] can be used, assuming the critical thickness is a fitted parameter:

$$
R(t)=\theta\left(t-t_{\mathrm{cr}}\right)\left(1-\frac{t}{t_{\mathrm{cr}}}\right)
$$

where $\theta\left(t-t_{\mathrm{cr}}\right)=1$ if $t>t_{\mathrm{cr}}$ and 0 if $t<t_{\mathrm{cr}}$. The relaxation values found from the experimental RSMs for reflection 224 (several RSMs were presented in Fig. 2) and reflection 404 (RSMs not shown here) for films of various thickness were fitted according to (9), the value of $t_{\mathrm{cr}}$ was found to be about $370 \mathrm{~nm}$ (Fig. 4). It is significantly larger than the value $19 \mathrm{~nm}$ for this system by Mathews and Blakeslee and makes evident that complex processes of dislocation nucleation and multiplication are here of primary importance [9].

The future development of the theory of X-ray diffraction from partly relaxed systems should contain dynamical

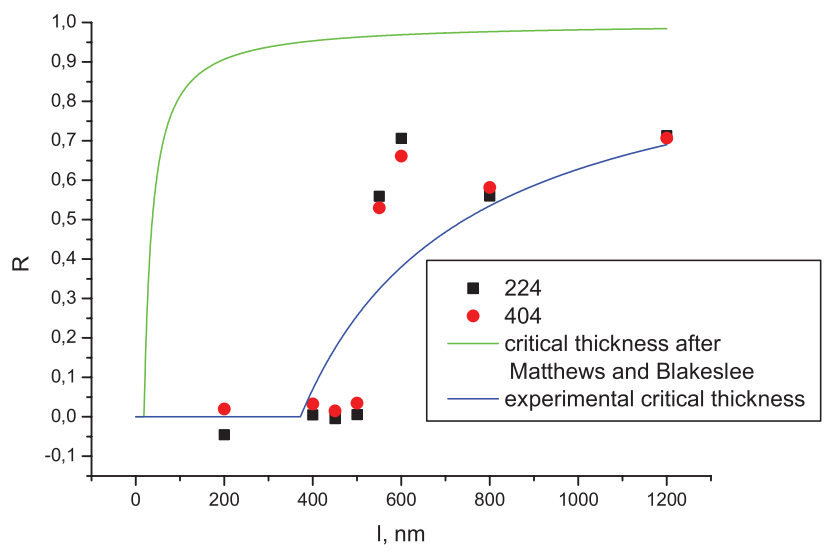

Figure 4 (online color at: www.pss-a.com) Dependence of relaxation on film thickness: experimental data, dependence from Ref. [8], and the same dependence with $t_{\mathrm{cr}}$ as fitted parameter. 
theory for coherent diffraction and Kaganer's theory for diffuse diffraction as limiting cases. It must have the ability to describe intermediate situations as well since they can be observed in the experiment. A progress in this direction was gained in the recent works of Kaganer and Sabelfeld [10], also the interest to the application of the statistical dynamical diffraction theory to the partly relaxed systems increased in last decade [11]. The development of a self-consistent uniformly suitable theory for the arbitrary relaxation degree describing simultaneously coherent and diffuse scattering of X-rays on partly relaxed systems will be a subject of forthcoming investigations.

\section{References}

[1] A. Benediktovich and I. Feranchuk, Phys. Status Solidi A 206, 1695 (2009).
[2] S. Danis, V. Holy, J. Stangl, and G. Bauer, Europhys. Lett. 82, 66004 (2008).

[3] U. Pietsch, V. Holy, and T. Baumbach, X-ray Scattering: From Thin Films to Lateral Nanostructures (Springer-Verlag, Heidelberg, 2004), p. 111.

[4] T. Sasaki, H. Suzuki, A. Sai, J.-H. Lee, M. Takahasi, S. Fujikawa, K. Arafune, I. Kamiya, Y. Ohshita, and M. Yamaguchi, Appl. Phys. Express 2, 085501 (2009).

[5] R. Kuzel, Z. Kristallogr. 222, 136 (2007).

[6] V. M. Kaganer, R. Köhler, M. Schmidbauer, R. Opitz, and B. Jenichen, Phys. Rev. B 55, 1973 (1997).

[7] P. M. Mooney, Mater. Sci. Eng. R17, 105 (1996).

[8] S. M. Hu, J. Appl. Phys. 69(11), 7901 (1991).

[9] R. Beanland, J. Appl. Phys. 72(9), 4031 (1992).

[10] V. M. Kaganer and K. K. Sabelfeld, Phys. Rev. B 80, 184105 (2009).

[11] P. K. Shreeman and R. J. Matyi, J. Appl. Cryst. 43, 550 (2010). 